\title{
Surface topography of CVD-W coatings on graphite substrate with a PVD-Si intermediate layer after thermal fatigue testing
}

\author{
X.M. Zhao ${ }^{a}$, J.P. Song ${ }^{b}$, Y. Yu ${ }^{b}$, Z.G. Zhuang ${ }^{c}$, B.Z. Sun ${ }^{a_{11}}$, Y. Qi ${ }^{a, d, 2}$ \\ ${ }^{a}$ Institute of Materials Physics and Chemistry, Northeastern University, Shenyang, 110819, China \\ ${ }^{b}$ Xiamen Honglu Tungsten Molybdenum Industry Co. Ltd., Xiamen, 361021, China \\ ${ }^{c}$ China National R\&D Center for Tungsten Technology, Xiamen Tungsten Co. Ltd., 361026 Xiamen, \\ PR China \\ ${ }^{d}$ Key Laboratory for Anisotropy and Texture of Materials, Ministry of Education, Northeastern \\ University, Shenyang 110819, China
}

1 Corresponding author: Tel. +86-24-83683674 Fax: +86-24-83683674

E-mail address: bzsun@imp.neu.edu.cn (B.Z.Sun).

2 Corresponding author: Tel. +86-24-83683674 Fax: +86-24-83683674

E-mail address: qiyang@imp.neu.edu.cn (Y. Qi). 


\begin{abstract}
This work deals with the effects of thermal fatigue testing on the surface topography of W coatings on graphite substrate with Si intermediate layers, where W coatings were deposited by chemical vapor deposition (CVD-W coatings) and Si intermediate layers were prepared by physical vapor deposition (PVD-Si layers). The surface topography of samples both as-deposited and after thermal fatigue testing were characterized by X-ray diffraction (XRD) and scanning electron microscope (SEM). Comparative studies show that CVD-W coatings have so excellent thermal conductivity that electronic beams are only absorbed by a thin layer on the coating surface. The absorbed thermal loads give rise to the melting of the pyramid-like $\mathrm{W}$ grain tips and the formation of shallow melting pools. Meanwhile, the size of the recrystallized grains on the surface is much smaller than those of the initial grains after cooling. Since the fine recrystallized grains can undoubtedly suppress the crack propagation, no visible failure was found in sample after thermal fatigue testing.
\end{abstract}

Keywords: Thermal fatigue testing, surface topography, CVD-W coatings, shallow melting pools, recrystallized grains 


\section{Introduction}

As one of the most important candidates as plasma facing material (PFM) for the International Thermonuclear Experimental Reactor (ITER), tungsten (W) has excellent physical properties, such as a high melting point (3773K) and high thermal conductivity $(235 \mathrm{~W} /(\mathrm{m} \cdot \mathrm{K})$ at $273 \mathrm{~K}-373 \mathrm{~K})$, low retention of tritium and a low erosion rate under plasma loading [1]. However, the main disadvantage of $\mathrm{W}$ is its brittleness, especially recrystallization brittleness [2]. In addition, the ductile to brittle transition temperature (DBTT) is high (approximately 400 WC), leading to difficulties in forming and machining. Consequently, on the one hand, to make tungsten ductile, it is necessary to decrease the DBTT by reducing the strain rate [3] or refining the grain size [4]. On the other hand, in order to apply and explore tungsten materials for repairing or updating the plasma facing components (PFCs) of the first wall and divertor in existing or future tokomaks, a promising and feasible method is that a thin $\mathrm{W}$ coating is deposited on a heat sink (copper and its alloys) or structural materials (steel). Unfortunately, there is a thermal expansion mismatch between the substrate, such as copper and steel. The coefficient of thermal expansion (CTE) of $\mathrm{Cu}$ and steel is about $17 \times 10^{-6} / \mathrm{K}$ and $12 \times 10^{-}$ ${ }^{6} / \mathrm{K}$ respectively, and $5 \times 10^{-6} / \mathrm{K}$ for $\mathrm{W}$. Such a coating can result in severe interface stress and failure of PFCs [5]. Hence the performance of PFCs is not only dependent on the W coating but also on the substrate materials and the bonding between them.

At present, to improve the thermal expansion mismatch, one method is to use functionally graded materials (FGMs) as an interlayer. For example, using W-Cu FGMs can mitigate the CTE between the $\mathrm{W}$ coating and $\mathrm{Cu}$ substrate [6-8]. These PFCs have shown good performance in resisting the heat flux loads [9-11]. Another method is to use metal $(\mathrm{Ti})$ or nonmetal $(\mathrm{Si}$ and $\mathrm{SiC})$ as an interlayer if the graphite or the carbon 
fiber reinforced composite (CFC) is chosen as substrate. For instance, when Si acts as the interlayer, an excellent option is the combination of $\mathrm{W}$ coating prepared by chemical vapor deposition (CVD-W coating) and Si interlayer deposited by physical vapor deposition (PVD-Si interlayer). It can endure the thermal fatigue tests with an absorbed power density of $4.62 \mathrm{MW} / \mathrm{m}^{2}$, loading cycles of $5 \mathrm{on} / 25$ off and thermal fatigue cycles number of 200, and that no catastrophic failure was found in the CVD-W coatings [11]. Compared to the $\mathrm{Cu}$ substrates and the FGMs or Ti interlayers, the graphite substrates and the PVD-Si interlayers have significant advantages, such as lowcost and simple preparation. Meanwhile, the deposited PVD-Si interlayer can avoid the reaction between graphite substrates and W coatings. However, after thermal fatigue testing, the surface topography and the crystalline states of CVD-W coatings have not been unclear yet. The microstructural information is associated closely to the physical properties. In this work, phase composition and surface topography of the CVD-W/PVD-Si/Graphite sample are investigated and discussed particularly, which can provide the interpretation why the CVD-W/PVD-Si/Graphite materials can show excellent performance under electron beam loadings.

\section{Experimental}

A CVD-W coating was produced subsequently by using the source gas, $\mathrm{WF}_{6}$, with a purity of 99.99 wt.\% provided by Xiamen Tungsten Co., Ltd, China. The purity of the carrier gas, $\mathrm{H}_{2}$, was 99.999 wt.\% (Linde Gas Xiamen Co., Ltd, China). An in-house made CVD-W apparatus was used in the experiments. The molar ratio of $\mathrm{WF}_{6}$ and $\mathrm{H}_{2}$ was $1: 3$, and the pressure of the gas mixture was 1 atmosphere. As described elsewhere [11], the thermal fatigue testing were performed on a $60 \mathrm{~kW}$ electron-beam material 
testing apparatus (EMS-60) located in Southwestern Institute of Physics (SWIP), China. The device provided an electron beam with a maximum power $60 \mathrm{~kW}$. The acceleration voltage of electron beam was in the range of $90-150 \mathrm{kV}$. The maximum electron current was $400 \mathrm{~mA}$. The cycle of the electron beam is $5 \mathrm{on} / 25$ off and the cycle numbers are 200. The electron beam fatigue loading was applied to a vertical W surface and focused to a diameter of approximately $1 \mathrm{~mm}$. It should be noted that there is no any compositional change due to thermal fatigue testing.

Phases compositions of the samples were identified by X-ray diffraction (XRD) results. A Rigaku-D/max-A diffractometer was employed for the XRD studies of the CVD-W coatings at an operating voltage of $30 \mathrm{kv}$. These measurements were performed using $\mathrm{CuK}_{\alpha}$ radiation (wavelength $\lambda_{\mathrm{K} \text { 龱 }}=1.5406 \AA$ ) in the $2 \theta$ range of $20-80[$. The scanning electron microscope (SEM) observations were performed using Hitachi S3400 and SU8010, prior to which the SEM samples were ground, polished, and chemically etched. The etching was performed with a 5\% $\mathrm{NaOH}, 5 \% \mathrm{~K}_{3}\left[\mathrm{Fe}(\mathrm{CN})_{6}\right], 90 \% \mathrm{H}_{2} \mathrm{O}$ solution at room temperature. For simplicity and the sake of discussion, as-deposited sample and the sample after thermal fatigue testing were defined as \#1 and \#2 samples, respectively.

\section{Results and discussion}

Fig.1 (a) and (b) depict the surface topography of as-deposited sample (\#1 sample) and the sample after thermal fatigue testing (\#2 sample), respectively. In the \#1 sample, the pyramid-like grains can be observed clearly, and that the edges of the pyramid-like grains were very sharp and the of the grains tips were removed. However, compared to the \#1 sample, the edges of the most of pyramid-like grains were smoother and the grain 
sizes were larger in the \#2 sample. Fig.1 (c) is the enlarged image of fig.1 (b), from which it can be observed that the pyramid-like grain tips were melted, as marked by red circle. From the amplification of the tip (shown in fig.1 (d)), it is visible that some square-like melting pools have been formed at the top of the grains. The depth of the pools varies from 10 to $30 \mu \mathrm{m}$ and the side lengths of the square-like pools were in range from 10 to $30 \mu \mathrm{m}$. The grains near the pools were different from the others, as shown in fig.1 (c). More importantly, the fine pyramid-like grains can be noticed in the pools, as marked by yellow arrow in fig.1 (d).

In order to understand the formation mechanism of melting pools and fine pyramid-like grains, a comprehensive observation of surface and cross-section of the \#2 sample was made. Fig.2 (a) demonstrates the low-magnification SEM images of outer walls of the melting pools at the top of pyramid-like grains. The etching triangular pyramid-like protrusions can be found on outer walls of the pyramid-like grains. Fig.2 (b) shows the amplification of rectangle b region in fig.2 (a), from which it can be verified that some smaller protrusions are located in the upper edges of outer wall, whereas, the larger protrusions are in the lower-middle parts, as marked by red rectangle $\mathrm{c}$ and $\mathrm{d}$. To some extent, the size of the protrusions reflects the size of the fine grains. As a consequence, the region marked by red rectangle $\mathrm{c}$ is called the fine grained region and the region marked by red rectangle $d$ is called the coarse grained region. Obviously, the fine grained regions are near the surface, inversely, the coarse grained regions are far away from the surface. From the enlarged parts of rectangle c and d (shown in fig.2 (c) and (d)), it can be observed that the protrusions are sparse in the coarse grained regions. The side lengths of protrusions in the coarse grained regions are about five times as those in the fine grained regions. Undoubtedly, for these fine grains outer walls 
of melting pools and fine pyramid-like grains in melting pools, their formation is owing to the process of the melting and the recrystallization during thermal fatigue testing. Fig.2 (e) is the enlarged image of rectangle e in fig.2 (a). Obviously, the side parts of outer walls are composed of coarse grains, suggesting that the range of coarse grained regions is larger. It demonstrated that only very thin $\mathrm{W}$ layer can be melt during thermal fatigue testing. Owing to the small energy of electron beam, only very small amounts of W can be eroded and the transpiration cooling should not be involved in this process. Fig.2 (f) shows the SEM image of the cross-section of the CVD-W coatings. With the columnar crystal structures, the coatings can be helpful to avoid coating flaking under high heat loads [9]. The cross-section of the surface grains has the characteristics of arch. It is verified that the microstructure of the grains near the surface are different from the others after thermal fatigue testing. These fine grained regions described in fig.2 (c) are corresponding to the region marked by the arrows in fig.2 (f).

Fig.3 (a) and (b) indicate the XRD patterns taken from the surfaces of the \#1 and the \#2 samples, respectively. For the \#1 sample, the relative peak intensities are approximately well consistent with those in the standard PDF card (JCPDS No. 040806) of $\mathrm{W}$, in which the strongest peak is assigned to $\mathrm{W}(110)$. Whereas, the strongest peak is W(200) in the \#2 sample, which is similar as reported in the literature [12]. It suggests that the surface grains grow dominantly along $<100>$ orientation after the thermal fatigue testing. This is mainly because the process of melting and resolidification change the surface topography and orientations of the grains in shallow melting zone.

Fig.4 shows the schematic diagrams of the surface topography variation of the CVD-W coating during the thermal fatigue testing. The shape of cross-section of as- 
deposited CVD-W coatings was triangle (fig.1 (a) and fig.4 (a)). The different positions of grains have different absorption of the heat loads. With the heat transfer from up to down, the top of the grains can undoubtedly absorbed more heat than the inside and the bottom. Therefore, the different positions of grains have different temperature owing to the different absorption of the heat loads during the thermal fatigue testing. As shown in fig.4 (b), the darker the color is, the higher the temperature is. With increasing heat flux cycles, when the temperature of surface ascends to the melting point of $\mathrm{W}$ (3773K), the pyramid-like grain tips will firstly began to melt and form melting pools at the top of the grains, as displayed in fig.1 (c) and fig.4 (c). The other positions of the grain surface absorbed less heat than the top, as a result, only the area about $10 \mu \mathrm{m}$ from the surface was melted. The area could be called the shallow melting zone. When the temperature of the surface drops rapidly during the cooling stage, the recrystallization will occur. It is well known that the recrystallization grain sizes depend on the supercooling degree of solidification. The grains exposed to the surface have the larger supercooling degree during the cooling stage, so the fine grained region emerge from the shallow melting zone, as shown in fig.2 (b), (d) and fig.4 (d). Likewise, the fine pyramid-like grains will also appear in shallow melting pools during the resolidification, as indicated in fig.4 (d) and (e).

After the thermal fatigue testing, no catastrophic failures were observed in these samples, which were owing to the following factors. Firstly, $\mathrm{W}$ has so excellent thermal conductivity that electronic beams were only absorbed by the coating surface and the most of energy in electronic beams can be transmitted to the bottom of the grains. Secondly, the fine grained regions formed during the cooling stage could be a protection layer to protect the CVD-W coatings from destruction because fine grains have higher 
strength, hardness, plasticity and toughness. The finer the grains are, the harder the crack propagation is. Finally, the CTE of substrate graphite $\left(1 \times 10^{-6} / \mathrm{K}\right.$ perpendicular to $c$-axis) is closer to $\mathrm{W}\left(5 \times 10^{-6} / \mathrm{K}\right)$ than $\mathrm{Cu}\left(17 \times 10^{-6} / \mathrm{K}\right)$. Therefore, compared to $\mathrm{Cu}$, using graphite as the substrate with CVD-W coatings could have no crack during the thermal fatigue testing. The CTE of interlayer $\mathrm{Si}\left(2.5 \times 10^{-6} / \mathrm{K}\right)$ is between $\mathrm{W}$ coatings and graphite substrates, which can mitigate the CTE mismatch between the $\mathrm{W}$ coatings and graphite substrates.

Although the melting has occurred during the thermal fatigue testing, the SEM characterization in our work can indicate that the melting and resolidification occurs only in the range of a thin $\mathrm{W}$ layer. Compared to flat surface, the scraggly surface morphology will absorb more thermal loads in unit area. The melting and recrystallization of a thin $\mathrm{W}$ layer result mainly from the heat accumulation on coating surface. Meanwhile, owing to the scraggly surface morphology, the possibility of lateral heat transfer will be reduced, which contributes to the heat accumulation on coating surface. On condition that the surface of CVD-W coating is relatively flat, the heat accumulation can be transferred instantaneously and the melting and recrystallization can hardly occur. Therefore, the surface morphology of CVD-W coatings is crucial to withstand the high heat load. The preparation of flat W coatings by means of CVD method, which is the key to the question obviously, is our next research emphasis.

\section{Conclusions}

By means of XRD and SEM, the surface topographies of the two kinds of CVDW/PVD-Si/graphite samples were characterized. The surface topography of asdeposited sample possesses the characteristic of pyramid-like grains. For the sample 
after thermal fatigue testing, the pyramid-like grain tips undergo a process of melting and resolidification. In this process, the CVD-W coatings have so excellent thermal conductivity that thermal loads are only absorbed by a thin W-layer on coating surface, which leads to the appearance of shallow melting pools. Meanwhile, the increase of undercooling results in the formation of fine grains. The described surface features can restrain the crack propagation and strengthen the coatings.

\section{Acknowledgements}

Financial support for this work was provided by the Fundamental Research Funds for the Central Universities (Grant No. L1502037) and the National Science Foundation of China (Grant No. 51172040)

\section{References}

[1] N. Ohno, S. Kajita, D. Nishijima, S. Takamura, Surface modification at tungsten and tungsten coated graphite due to low energy and high fluence plasma and laser pulse irradiation, J. Nucl. Mater. 363-365 (2007) 1153-1159.

[2] J. Reiser, M. Rieth, B. Dafferner, A. Hoffmann, Charpy impact properties of pure tungsten plate material in as-received and recrystallized condition (1 h at 2000 degrees C (2273 K)), J. Nucl. Mater. 442 (2013) S204-S207.

[3] P. Gumbsch, Brittle fracture and the brittle-to-ductile transition of tungsten, J. Nucl. Mater. 323 (2003) 304-312.

[4] J. Reiser, J. Hoffman, U. Jantsch, M. Klimenkov, S. Bonk, C. Bonnekoh, M. Rieth, A. Hoffmann, T. Mrozek, Ductilisation of tungsten (W): On the shift of the brittle- 
to-ductile transition (BDT) to lower temperatures through cold rolling, Int. J. Refract. Met. Hard. Mater. 54 (2016) 351-369.

[5] T. Hirai, A. Kreter, J. Linke, J. Malzbender, T. Ohgo, V. Philipps, G. Pintsuk, A. Pospieszczyk, Y. Sakawa, G. Sergienko, T. Tanabe, Y. Ueda, M. Wada, Critical heat flux loading experiments on CVD-W coating in the TEXTOR tokamak, Fusion Eng. Des. 81 (2006) 175-180.

[6] J. Chapa, I. Reimanis, Modeling of thermal stresses in a graded Cu/W joint, J. Nucl. Mater. 303 (2002) 131-136.

[7] G. Pintsuk, S.E. Brunings, J.E. Doring, J. Linke, I. Smid, L. Xue, Development of W/Cu - functionally graded materials, Fusion Eng. Des. 66-68 (2003) 237-240.

[8] Z. Zhou, S. Song, J. Du, Z. Zhong, C. Ge, Performance of W/Cu FGM based plasma facing components under high heat load test, J. Nucl. Mater. 363-365 (2007) 1309-1314.

[9] Y. Lian, X. Liu, Z. Xu, J. Song, Y. Yu, Preparation and properties of CVD-W coated W/Cu FGM mock-ups, Fusion Eng. Des. 88 (2013) 1694-1698.

[10] J.P. Song, Y. Yu, Z.G. Zhuang, Y. Lian, X. Liu, Y. Qi, Preparation of W/Cu functionally graded material coated wth CVD-W for plasma-facing components, J. Nucl. Mater. 442 (2013) S208-S213.

[11] J.P. Song, Y. Lian, Y. Lv, J. Liu, Y. Yu, X. Liu, B. Yan, Z. Chen, Z.G. Zhuang, X. Zhao, Y. Qi, Development of CVD-W coatings on CuCrZr and graphite substrates with a PVD intermediate layer, J. Nucl. Mater. 455 (2014) 531-536.

[12] J. Du, Z. Li, G. Liu, H. Zhou, C. Huang, Surface characterization of CVD tungsten coating on molybdenum substrate, Surf. Coat. Tech. 198 (2005) 169-172. 


\section{Captains of figures:}

Fig.1. The SEM images showing the surface topography of CVD-W coatings: (a) asdeposited, (b) after the thermal fatigue testing, (c) the enlarged image of (b), (d) the amplification of melting pool in (c).

Fig.2. The SEM images of the surface and the cross-section of CVD-W coatings after the thermal fatigue testing: (a) the morphology of outer wall of melting pools at the top of pyramid-like grains, including (b) the top of (a), including coarse grained region and fine grained region the right side of (a), (c) and (d) is (b) enlarged image of coarse grained region and fine grained region in (b), respectively. The locations are indicated as "b” "e” in (a), “c” "d" in (b).

Fig.3. The XRD patterns taken from the surface of (a) the \#1 sample and (b) the \#2 sample.

Fig.4. The schematic drawing of the surface topography variation of the CVD-W coating during the thermal fatigue testing and the cooling stage: (a) the as-deposited CVD-W coatings, (b) shallow melting occurring at the surface, (c) the melting pools emerging at the top of grain, (d) formation of the grains in the melting pools and the shallow melting zone changing into fine grained region during the cooling stage, (e) as the time goes, pyramid-like grains growing. 
Figures
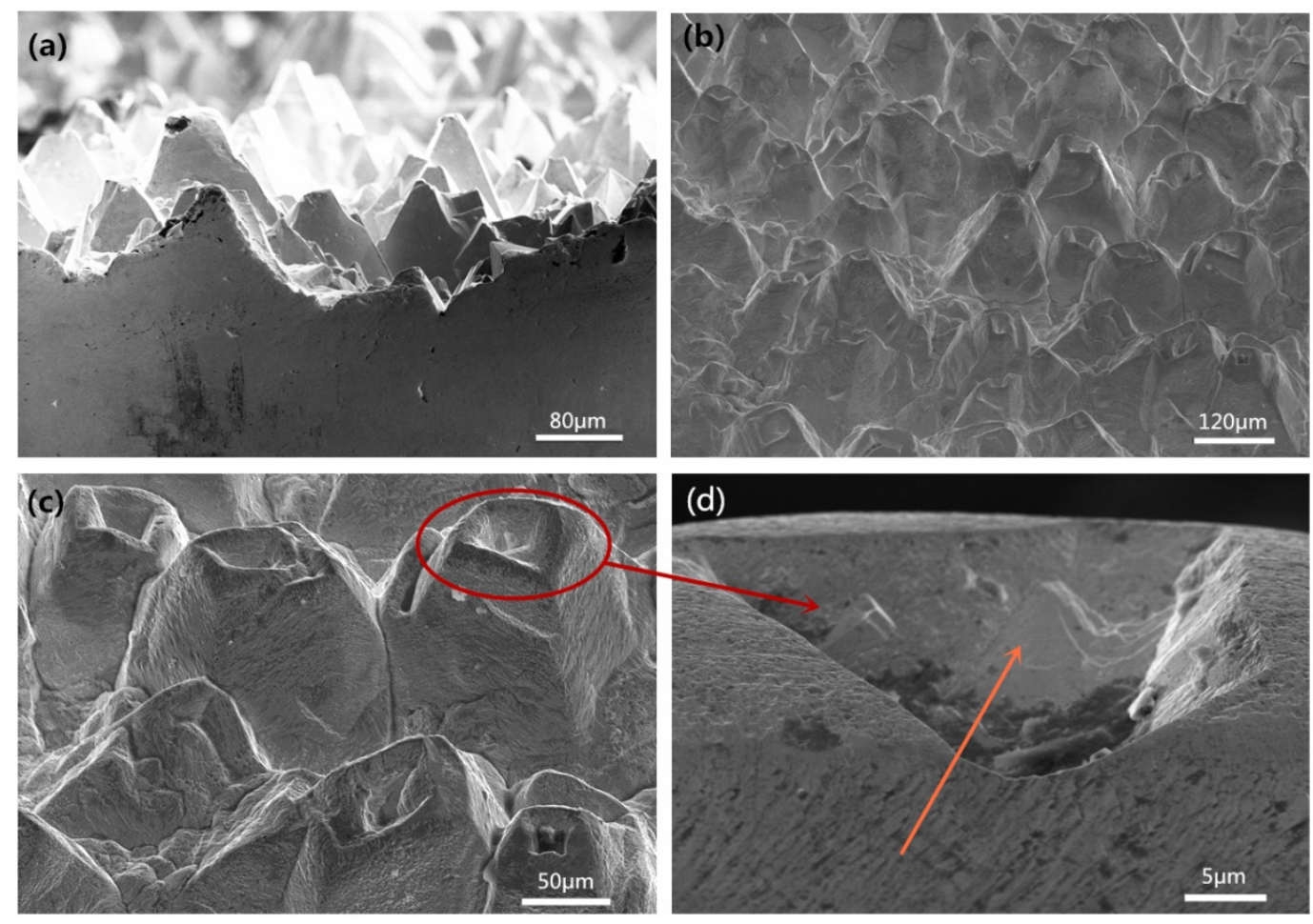

Fig.1 

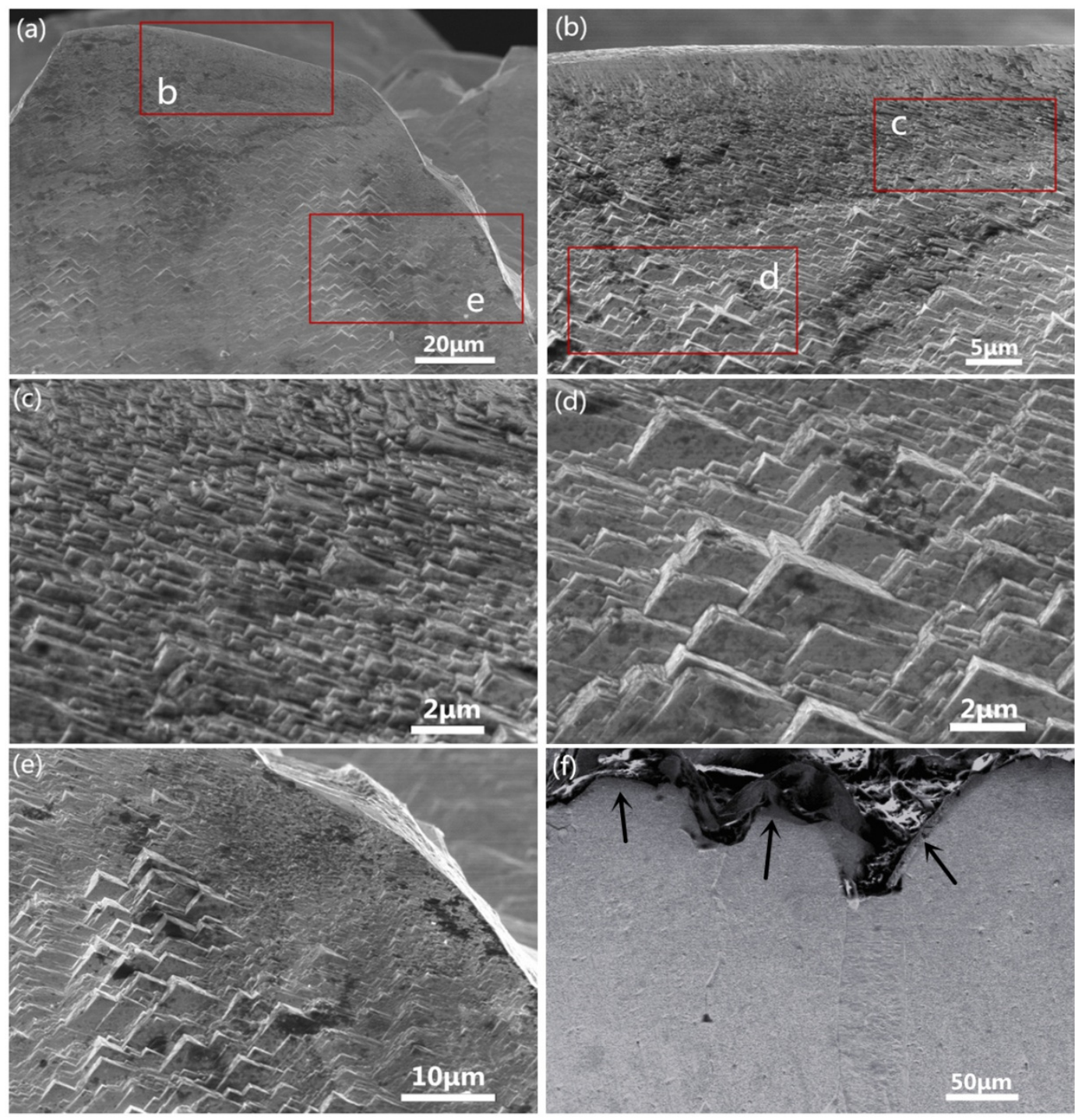

Fig.2 

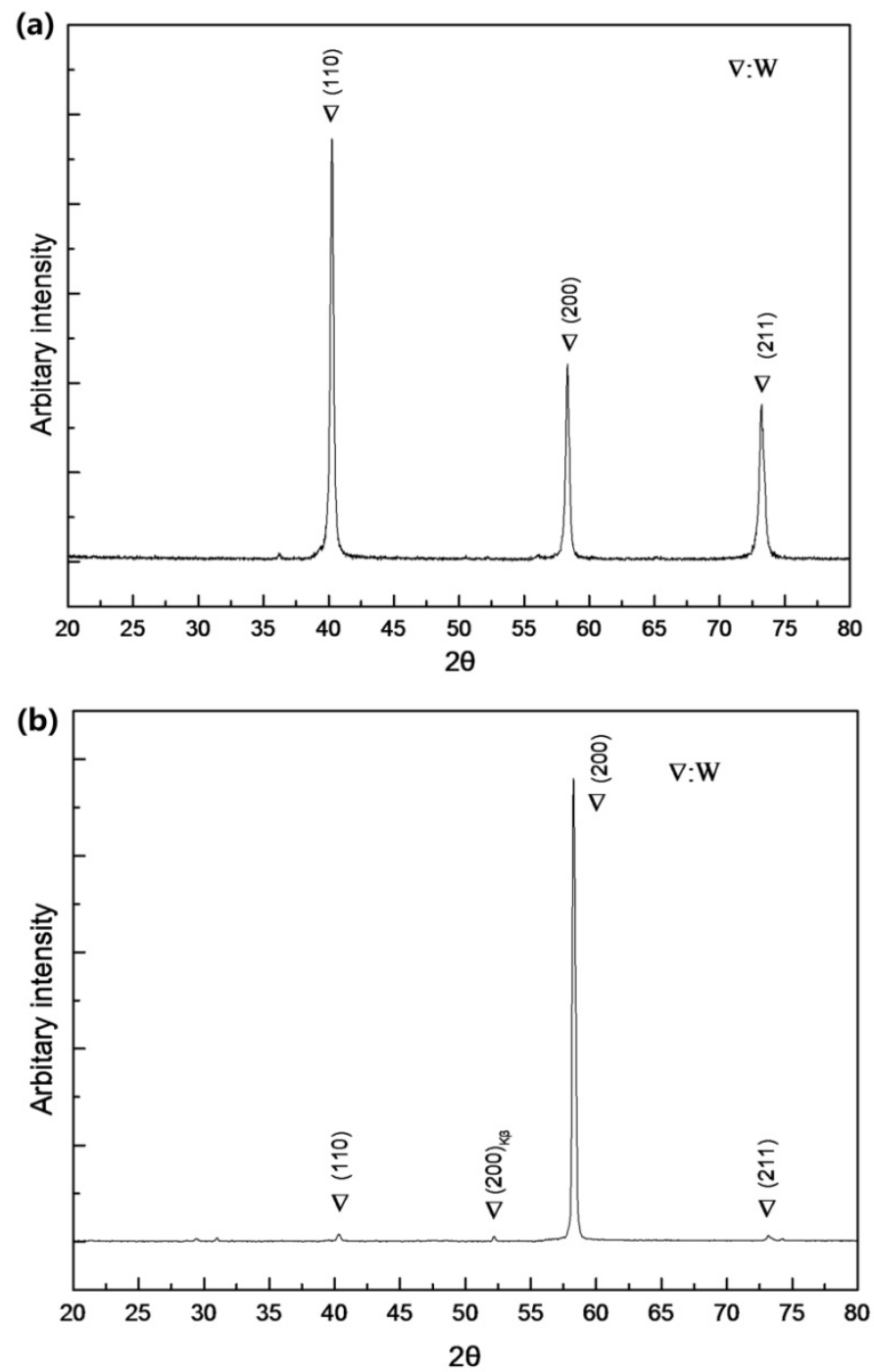

Fig. 3 

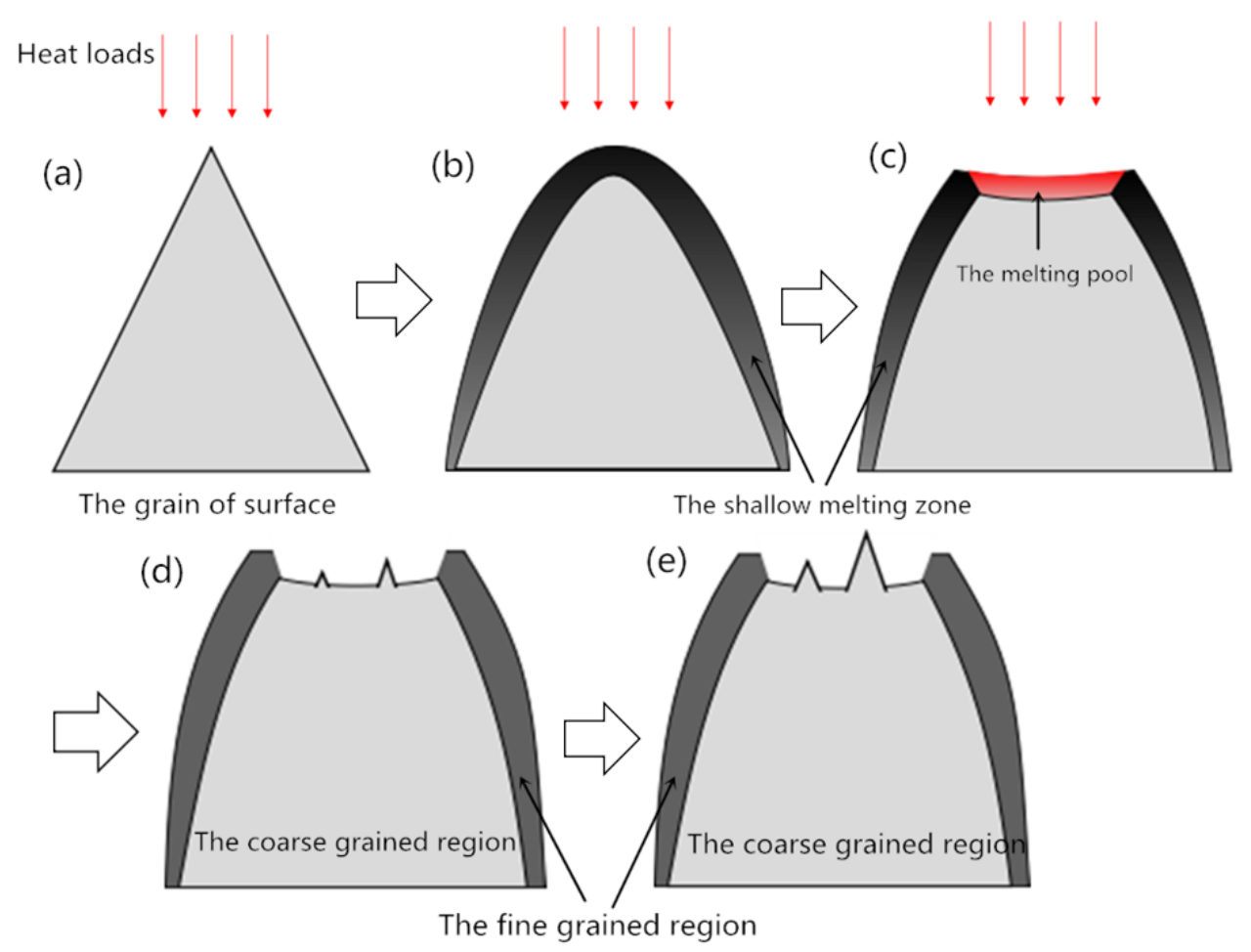

Fig.4 